International Journal of Network Security \& Its Applications (IJNSA), Vol.4, No.3, May 2012

\title{
ENERgY CONSTRAINED RELIABLE ROUTING OPTIMIZED Cluster heAd PROTOCOL FOR MULTIHOP UNDER WATER ACOUSTIC SENSOR NETWORKS
}

\author{
Padmavathy.T.V ${ }^{1}$, Gayathri. $V^{2}$, Indhumathi. $V^{3}$, Karthiga.G ${ }^{4}$ \\ Department of Electronics and Communication Engineering \\ R.M.K Collge of Engineering, Chennai, India \\ tvpssn@gmail.com ${ }^{1}$, svgayu@gmail. $\mathrm{com}^{2}$, indumathivelu9o@gmail. $\mathrm{com}^{3}$, \\ gunasekhar.karthika@gmail. $\mathrm{com}^{4}$
}

\begin{abstract}
Underwater acoustic sensor network is an emerging technique consisting of sensor nodes, and AUVs all working together to sense various phenomenon, converts the sensed information into digital data, store the digital data and communicate to the base stations through the intermediate nodes. Also Underwater Acoustic Sensor Networks are playing a main role in ocean applications. Unfortunately the efficiency of underwater Acoustic Sensor Networks is inferior to that of terrestrial sensor networks due to the long propagation delay, narrow bandwidth and high error rates. Also battery life and storage capacity of node is limited. Many routing protocols are proposed to improve the efficiency of Under Water Acoustic Sensor Networks. However their improvement is not enough, so there is a need of suitable routing protocol that consider all these limitations and makes communication in underwater network viable. In this paper, we propose a protocol called Reliable Routing Optimized Cluster Head (RROCH) protocol, a network coding approach for multihop topologies. We used performance metrics like packet delivery ratio, energy consumption, end-to-end delay and throughput of sensor nodes. LEACH, HMR-LEACH, LEACH-M are compared for their performance at different traffic conditions, number of nodes and depth. By analyzing our simulation results we found that RROCH protocol may be used for denser network with low traffic and HMR- LEACH protocol is suitable for higher traffic with less number of nodes.
\end{abstract}

\section{KEYWORDS}

Acoustic Sensor Networks, Buffer Length, Energy Consumption, NS-2.26, optimum cluster size

\section{INTRODUCTION}

The research of Under Water Acoustic Sensor Network (UWASN) is attracting due to their important under water application for military, emergency and commercial purpose. The underwater environment is much different from terrestrial environment. Due to the high dense salty water the EM wave cannot be transmitted for long distance in the ocean [1]. This is because of high attenuation and absorption effect in under water environment. To overcome this problem acoustic sensors can be used which will provide a better communication in under water environment. Even the optical signal gets scattered and absorbed by the underwater because it can't travel to a long distance in salty water. Due to scattering and adsorption of 
signal in under water communication the signal attenuated. Some of the challenges in under water communication are propagation delay, high bit error rate and limited bandwidth. In this harsh environment to provide low energy consumption we proposed a protocol to overcome the challenges in UWC.

The main objective of this paper is to minimize the power consumption and to improve the reliability of data transmission. Instead of transmitting data from source to sink directly if we use clustering method, it will lead better energy consumption in UWC. The UWSN has large number of sensor nodes. These nodes are grouped as several clusters based on the following costs.1) Energy level 2) Buffer length 3) Distance between the nodes. Each associate node in a cluster passes the data to a cluster head, and then the data is transmitted to sink through the cluster heads. The cluster head is selected in random manner in each transmission phase.

\section{RELATED WORK}

In this paper main challenge for the development of efficient networking solutions posed by the underwater environment are detailed and a cross-layer approach to the integration of all communication functionalities is suggested [1]. Network coding can improve throughput, robustness, complexity, and security [2]. In [3] this paper they proposed UWMAC, a transmitter-based CDMA MAC protocol for UWASNs that incorporates a novel closed-loop distributed algorithm to set the optimal transmit power and code length to minimize the nearfar effect. UW-MAC aims at achieving three objectives such as high network throughput, low channel access delay, and low energy consumption. Fatma Bouabdallah and Raouf Boutaba proposed UW-OFDMAC, a distributed Medium Access Control (MAC) protocol which provides low energy consumption and high bandwidth. A large number of closely spaced orthogonal sub-carrier signals carry data. The data is divided into several parallel data streams or channels, one for each sub-carrier [4]. The authors proposed a new multi-path routing algorithm, which taking the distance and energy into account to determine the transmission path. HMR-LEACH algorithm put the energy on the first priority when chooses the transmission path [5]. Based on analysis of energy consumption for LEACH in underwater channel, the authors proposed a novel scheme for cluster-head selection to ensure nodes energy load balance by considering residual energy of candidate nodes and the distance to SINK. Then it avoids choosing nodes with lower residual energy and bad position as cluster-heads and then provides the energy load's proportionality of sensor node [6].

In [7], they propose a novel energy efficient MAC protocol named NOGO-MAC (Node Grouped OFDMA MAC) which is based on orthogonal frequency division multiple accesses (OFDMA) and exploit the physical characteristic that propagation loss of acoustic wave depends on the distance more heavily at high frequency than at low frequency. In a wireless sensor network the sensor nodes are divided into several clusters according to the position of the sink. Maximal-energy method selects a head which contains the maximal energy of this cluster[8]. In [9] the authors investigate the selection of CHs in a distributed environment such as MANET. They derive new results on the algorithmic complexity of two variants of the $\mathrm{CH}$ selection In this paper, a novel, token-based medium access control (TMAC) solution for underwater acoustic broadcast is introduced. This TMAC solution avoids the need for synchronization and therefore underwrites successful communication [10]. Distance-Aware Collision Avoidance Protocol (DACAP) a non-synchronized protocol that allows a node to use different hand-shake lengths for different receivers so as to minimize the average handshake duration. It provides higher throughput than Slotted FAMA with similar power efficiency [11]. 
In this paper author proposed LEACH protocol, is one of the clustering routing protocols in wireless sensor networks. It can be used to enhance the performance of cluster-based wireless sensor networks in terms of lifetime and throughput [12]. Based on LEACH algorithm, the author proposed an improved HMR-LEACH algorithm (Hierarchical Multi-path RoutingLEACH), which improves election of cluster head and adopts multi-hop algorithm instead of one hop to transmission data. HMR-LEACH outperforms the LEACH algorithm and prolongs the life of the network dramatically [13]. In [14] this paper they propose the new cluster head selection protocol namely HEECH. This protocol selects a best sensor node in terms of energy and distance as a cluster head.

\section{Mechanism Of Reliable Routing Optimized Cluster Head PROTOCOL}

Operation of the RROCH protocol is divided into two phases, (i) the initialization phase and (ii) the data transfer phase is shown in Figure 1. During the initialization phase, clustering is done and cluster-heads are elected. During the data transfer phase, the nodes send their data periodically, at their scheduled times, to their $\mathrm{CHs}$. Finally, cluster-heads send their data to their neighbouring cluster head until the data reaches to the sink. The $\mathrm{CH}$ election is based on a energy level and buffer level that must be more than a calculated threshold

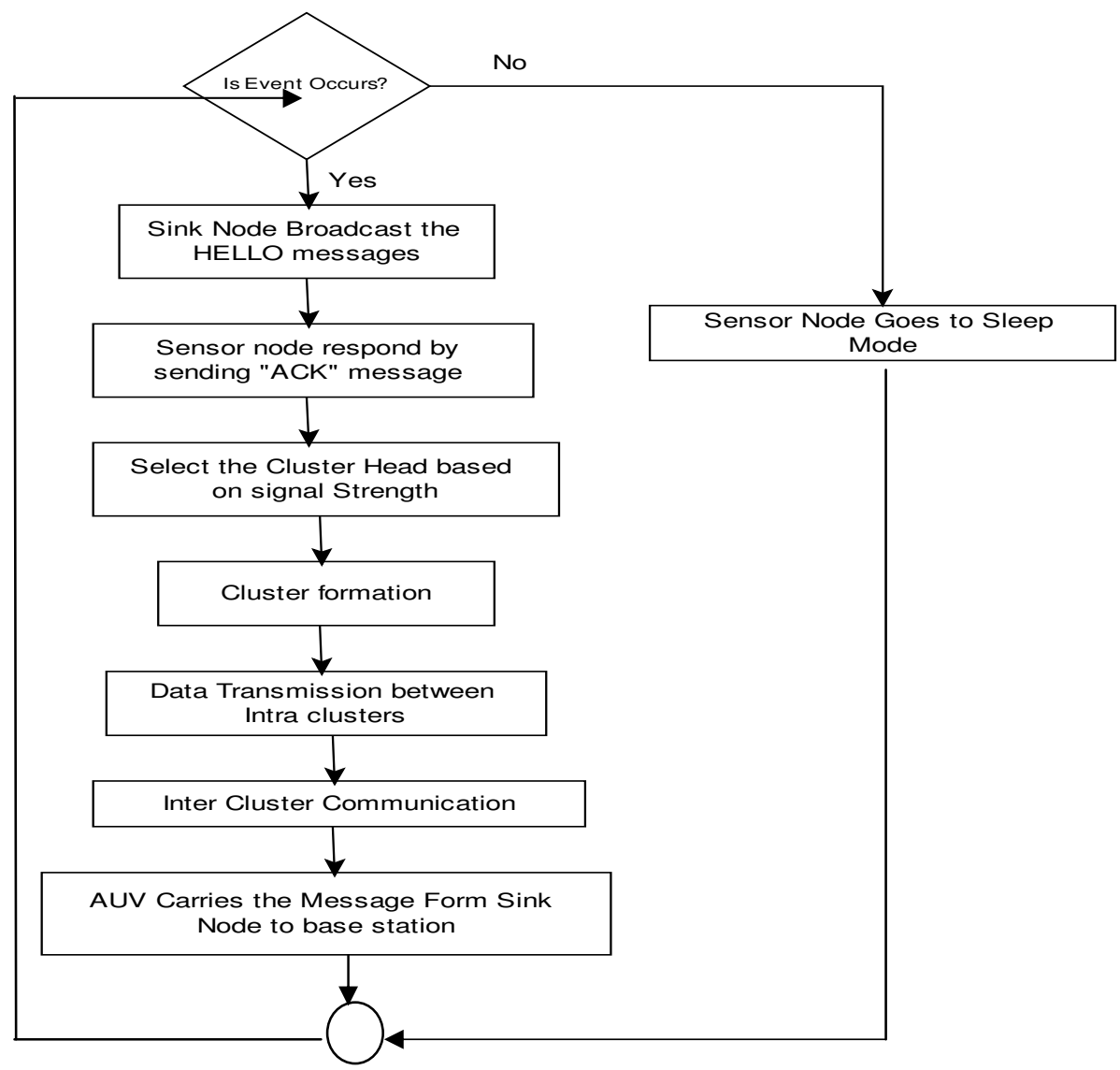

Figure 1. Mechanism of Reliable Routing Optimized Cluster Head Protocol 
International Journal of Network Security \& Its Applications (IJNSA), Vol.4, No.3, May 2012

\section{NETWORK MODEL}

1. All sensors are heterogeneous, predetermined and uniform randomly deployed within communication range of the sink node.

2. Every node is assumed to use the same, fixed power level for intra-cluster communication (e.g. broadcasting and communicate with $\mathrm{CH}$ ). For the outside cluster communication, $\mathrm{CHs}$ are capable of increasing its transmission power level to reach its sink node. And the sink nodes can also use power control to vary the amount of transmission power according to the distance to the desired recipient.

3. A sensor node monitors events or environmental conditions, such as temperature, pressure, and salinity.

4. Each sensor node has inherent ID (identification number) for identification purpose.

5. A cluster based network can be partitioned into disjoint clusters. Each cluster consists of one cluster head $(\mathrm{CH})$ and multiple associates (AS). Each $\mathrm{CH}$ collects data from its ASs and relays the processed data, e.g., the aggregated data to the base station (SINK).

6. In the whole network the OFDMA protocol is used. Each cluster use a different frequency, inter-cluster communication backbone is built by using OFDMA, ASs use Network Coding Algorithm and OFDMA protocol to send data to the neighbor cluster.

\section{Proposed RROCH Protocol}

The function of our protocol based on iterations referring to LEACH. Each iteration begins with a initialization phase when the cluster heads are selected and the clusters are organized, followed by a data transfer phase when the intra-cluster information are exchanged, the member nodes are chosen, and the merged data are transmitted to the inter cluster heads. Fig. 3 shows the time line for the iteration of RROCH protocol.

In this paper we consider the network scenario as node $N_{i}(1 \leq \mathrm{i} \leq 6)$ wants to broadcast its information to node $N_{j}(1 \leq j \leq 6, i \neq j)$ i.e., all the nodes in the cluster broadcast information to all the other nodes in the same cluster. Nodes in sensor clusters are divided into two types: cluster head and cluster member nodes. Assume that each node $N_{i}$ can successfully broadcast t of information to the corresponding cluster head within a given transmission range. The transmission range is the same for all nodes, while cluster head can reach every node in cluster by only one hop. All cluster member nodes have not the global topology information, while cluster head has. For each node, there exists at least one other node, such that the two nodes transmission range covers the entire cluster. Let $T$ denote the total number of transmissions required to finish information exchange process, and let $n$ denote the number of member nodes in the cluster. In under water acoustic wireless sensor networks, the total energy consumed can be represented as the sum of transmitter Energy, Receiver Energy, Sensing Energy and Computation Energy, this can be given as,

$E_{\text {total }}=E_{t x}+E_{r x}+E_{S}+E_{C}$ 


\subsection{Initiation of Transmission}

In the initialization phase a node starts a transmission after the initiation condition is true, the initiative decision is defined as follows

$$
\begin{aligned}
I & =1 \text { if } \beta=1 ; \text { otherwise } \\
& =0
\end{aligned}
$$

That is the initiation of transmission starts if the nodes detects an event $(\beta=1)$ otherwise the nodes goes to sleep mode if ( $\beta=0)$.

The two main function of the initialization phase are first one is to segregate the cluster nodes and sink nodes and the second one is to decide a transmission order of sensor nodes in same group.

\subsection{Initialization Phase}

In the beginning of an initialization phase, the sink node broadcasts a HELLO packet to the sensor nodes which are in the communication range of sink node. The HELLO packet format is given in Figure 2. The HELLO packet is contains a timestamp value that is time at which the HELLO message departed from the sink node, energy level and buffer length of the sink node. All sensor nodes receive $H E L L O$ packet within the maximum propagation delay of the sink node. When the sensor node receives HELLO packet, it shall response by using an acknowledgement message (ack). The ack message contains a timestamp value that is time at which the ack message received by the sensor node and it also has the signal strength. If each sensor nodes were to respond ack message immediately, the ack messages might result in collisions at the sink node. When transmitting the ack message to the sink node, sensor nodes use Linear Network Coding algorithm to avoid the collision, re-transmission and duplication of messages at the sink node. Using timestamp in each ack messages, the sink node can calculate the round trip time (RTT) and the distance between the sensor nodes and sink node very easily [7]. Sensor nodes are grouped by using clustering process.

\begin{tabular}{|l|l|l|l|l|}
\hline $\begin{array}{l}\text { Time Stamp } \\
(1 \text { byte })\end{array}$ & $\begin{array}{l}\text { Source ID } \\
(2 \text { byte })\end{array}$ & $\begin{array}{l}\text { Type of node } \\
(1 \text { Byte })\end{array}$ & $\begin{array}{l}\text { Energy Level } \\
(4 \text { byte })\end{array}$ & $\begin{array}{l}\text { Buffer Length } \\
(4 \text { byte })\end{array}$ \\
\hline
\end{tabular}

Figure 2. HELLO Packet format

\subsection{Underwater Channel Model}

In the underwater environment, acoustic channel attenuation of distance $d$ can be expressed as

$$
A(d)=d^{\lambda} \alpha^{d}
$$

Where $\lambda$ is the energy spreading factor ( 1 for cylindrical, 1.5 for practical, and 2 for spherical spreading). $x=10^{\alpha(f) / 10}$ is a frequency dependent term obtained from the absorption coefficient $\alpha(f)$. The absorption coefficient for the frequency range of interest is calculated according to Thorp's expression [6], 
International Journal of Network Security \& Its Applications (IJNSA), Vol.4, No.3, May 2012

$10 \log \left(\alpha(f)=0.11 \frac{f^{2}}{1+f^{2}}+44 \frac{f^{2}}{4100+f}+2.75 \times 10^{-4} f^{2}+0.003\right.$

in $\mathrm{dB} / \mathrm{km}$ for $f$ in $\mathrm{kHz}$.

\subsection{Energy Model}

Figure 3 shows the energy model for sensor node. In this work, we assume a simple model where the radio dissipates $E_{\text {elec }}=50 \mathrm{~nJ} / \mathrm{bit}$ bit to run the transmitter or receiver circuitry and $\varepsilon_{\text {amp }}=100 \mathrm{pJ} / \mathrm{bit} / \mathrm{m}^{2}$ for the transmit amplifier to achieve an acceptable $\frac{E_{b}}{N_{0}}$. We also assume that the packet size is $k=512$ bits

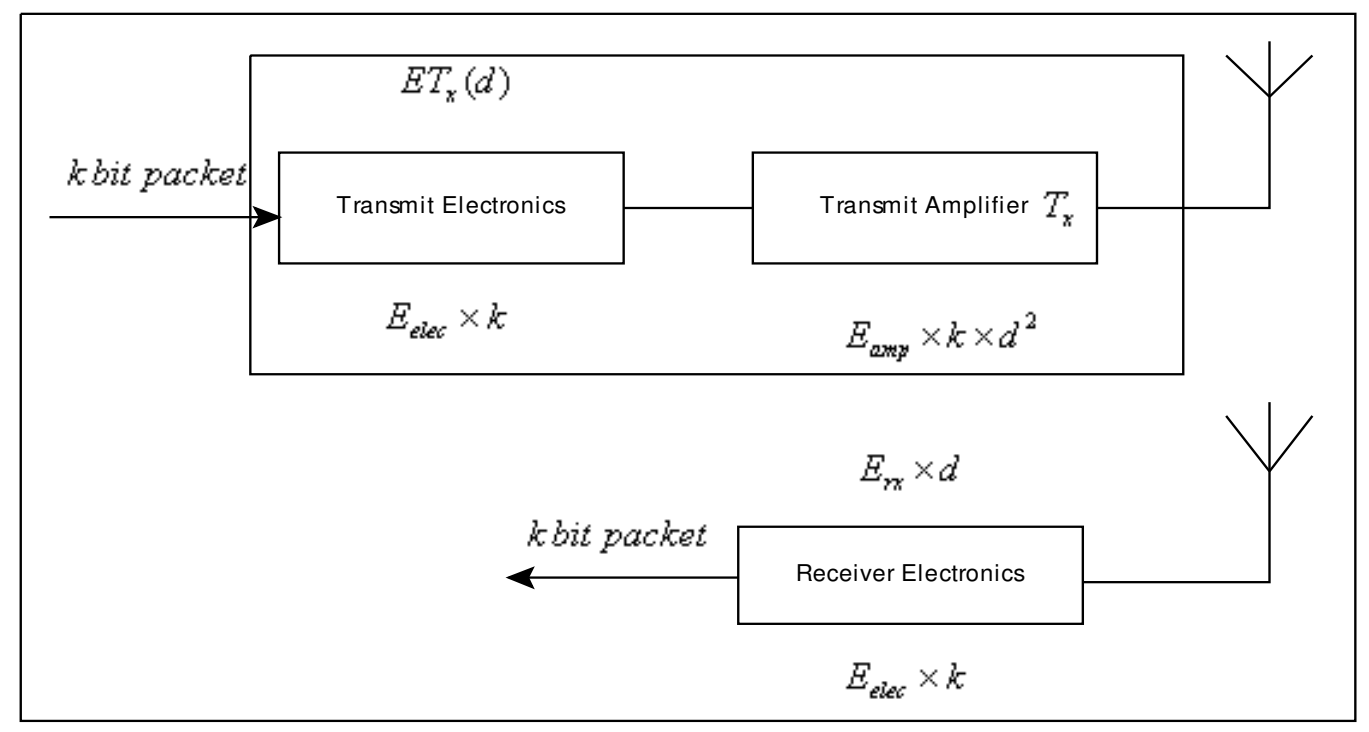

Figure 3. Energy Model

To transmit a data packet from one node to another over a distance $d$, the energy dissipation in underwater channel of each node is

$$
\begin{gathered}
E(d)=E_{t}(d)+E_{r}(d) \\
E_{t}(d)=l\left(E_{\text {elec }}+\varepsilon_{\text {amp }}\right)+P_{t} \times \frac{l}{\alpha \times B(d)} \\
E_{r}(d)=l\left(E_{\text {elec }}+E_{D A}\right)+P_{r} \times \frac{l}{\alpha \times B(d)}
\end{gathered}
$$

Where, $P_{t}$ and $P_{r}$ is transmit and receive power respectively, independent of distance rather depends on the complexity of the receive operations, $l$ is packet size; $B(d)$ is the bandwidth 
available and $\alpha$ is the bandwidth efficiency of the modulation in $\mathrm{bps} / \mathrm{Hz}, E_{\text {elec }}$ is the unit energy consumed by the electronics to process one bit of message, $\varepsilon_{\text {amp }}$ is energy consumed by amplifier, $E_{D A}$ is the energy for data aggregation.

\subsection{Energy Consumption during the Initialization Phase}

In this phase both the cluster head and non-cluster head nodes consumes energy. Initially it is assumed that all the sensor nodes have different amount of energy. In the election Phase, the cluster head first sends advertisement messages to all the non-cluster head nodes. Next the associate nodes receive the broadcasted messages from the different cluster heads and based on the received signal strength it chooses its own cluster head. The energy consumed by the cluster head is given by equation (8).

$$
E_{k}=N_{\text {asn }}\left(E_{\text {elec }}+\eta\left(E_{\text {elec }} T_{b} E_{t} \varepsilon_{\text {amp }}\right)\right)+l \varepsilon_{\text {amp }} d_{\text {snk }}^{2}
$$

Where, $N_{a s n}$ is number of associate nodes, $\eta$ is refers to us data aggregation ratio, $T_{b}$ is bit duration.

The energy consumed by the non-cluster head nodes is given by equation (9).

$$
E_{k i}=l E_{\text {elec }}+T_{b} P_{t} \varepsilon_{\text {amp }}
$$

From the equation (8) and (9) the total energy consumed by $k$ clusters is given by,

$$
E_{\text {total }}=\sum_{1}^{N} E_{k}+\sum_{1}^{N} E_{k i}
$$

The first part of the equation (10) gives the energy expended to receive the messages from $k$ clusters and the second part of the equation gives the energy expended to transmit the acknowledgement messages to the corresponding cluster head.

Placing few heterogeneous nodes in the network can bring three main benefits, the first one is to extend network lifetime that is the average energy consumption for forwarding a packet from the heterogeneous node to a base station will be much less than the energy consumed in homogeneous sensor networks, second is to improving reliability of data communication that is the heterogeneous sensor network can get much higher end-to-end delivery rate than the homogeneous sensor network and third one is to decrease the latency of data transmission that is the heterogeneous nodes can decrease the forwarding latency by using fewer hops to the Base station.

\subsection{Optimum Number of Clusters}

The optimum number of clusters can be found by setting the derivative of the total energy with respect to $k$ to zero. 
$\frac{d E_{\text {total }}}{d k}=0$

From equation (10) we obtained the total energy as given below,

$E_{\text {total }}=\left[N_{\text {asn }}\left(E_{\text {elec }}+\eta\left(E_{\text {elec }} T_{b} E_{t} \varepsilon_{\text {amp }}\right)\right)+l \varepsilon_{\text {amp }} d_{\text {snk }}^{4}\right]+l E_{\text {elec }}+T_{b} P_{t} \varepsilon_{\text {amp }}$

The optimum number of clusters can be obtained as follows

$\frac{d E_{\text {lotal }}}{d k}=\frac{\left[N_{\text {asn }}\left(E_{\text {elec }}+\eta\left(E_{\text {elec }} T_{b} E_{t} \varepsilon_{\text {amp }}\right)\right)+l \varepsilon_{\text {amp }} d_{\text {snk }}^{4}\right]+l E_{\text {elec }}+T_{b} P_{t} \varepsilon_{\text {amp }}}{d k}=0$

After simplification we obtain the optimum number of clusters which is given in equation (14)

$$
k_{\text {opt }}=\sqrt{\frac{N}{2 \pi}} \sqrt{\varepsilon_{\text {amp }}} \frac{M}{d_{\text {snk }}^{2}}
$$

\subsection{Cluster Head Selection}

The cluster head selection algorithm is a distributed algorithm similar to LEACH. In each iteration, all nodes organize themselves into $k$ local clusters. Using timestamp in each acknowledgement messages, the sink node can calculate the round trip time (RTT) and the distance between the sensor nodes and sink node very easily [7]. Sensor nodes are grouped by using clustering process.

\subsection{Analytical Model for Channel Utilization}

The effect of the characteristics of the underwater environment on the acoustic channel utilization efficiency as shown in Figure 4, which is defined as the net bit rate achievable on a link when considering packet retransmissions due to channel impairments, and provide guidelines for the design of routing solutions. When a random access technique is adopted to transmit a data packet in the shared acoustic medium a tradeoff between channel efficiency and link reliability occurs in fact, while the former increases the latter decreases with the increase of the packet size. Conversely, our routing solutions allow achieving two conflicting objectives, i.e., increasing the efficiency of the acoustic channel by transmitting a train of short packets back-to-back; and limiting the packet error rate by keeping the length of the transmitted packets short.

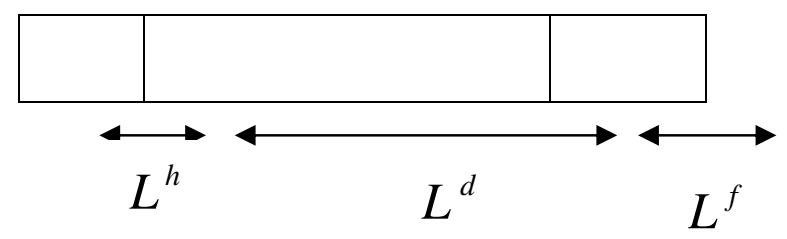

Figure 4. (a) 
$\mathrm{L}^{\mathrm{d}}$ - Payload of data packet

$L^{h}$ - Length of header bit

$L^{f}$ - Length of redundancy bit (FEC)

$L^{A}$ - Length of Acknowledgement Packet

Round Trip Transmission time $T_{r t t x}$ :

$T_{r t t x}=T_{t x}^{h}+T_{t x}^{d}+T_{t x}^{f}+2 T_{p}+T_{r x-t x}+T_{t x}^{A}$

$T_{r x-t x}$ is the time needed to process the packet and switch the circuitry from receiving to transmitting mode. The channel utilization efficiency is given by,

$\eta=\frac{L^{d}}{N_{t x} T_{r t t x}}$

$\eta=\frac{L^{d}}{N_{t x}\left[\mathrm{~T}_{\mathrm{tx}}^{\mathrm{h}}+\mathrm{T}_{\mathrm{tx}}^{\mathrm{d}}+\mathrm{T}_{\mathrm{tx}}^{\mathrm{f}}+2 T_{p}\left(T_{r x-t x}\right)+\mathrm{T}_{\mathrm{tx}}^{\mathrm{A}}\right]}$

Where $N_{T X}$ represents the average number of transmissions for the receiver to successfully decode a packet at a given packet error rate (PER) on the link.

$N_{T X}=(1-P E R) \sum_{n=1}^{\infty} n(P E R)^{n-1}$

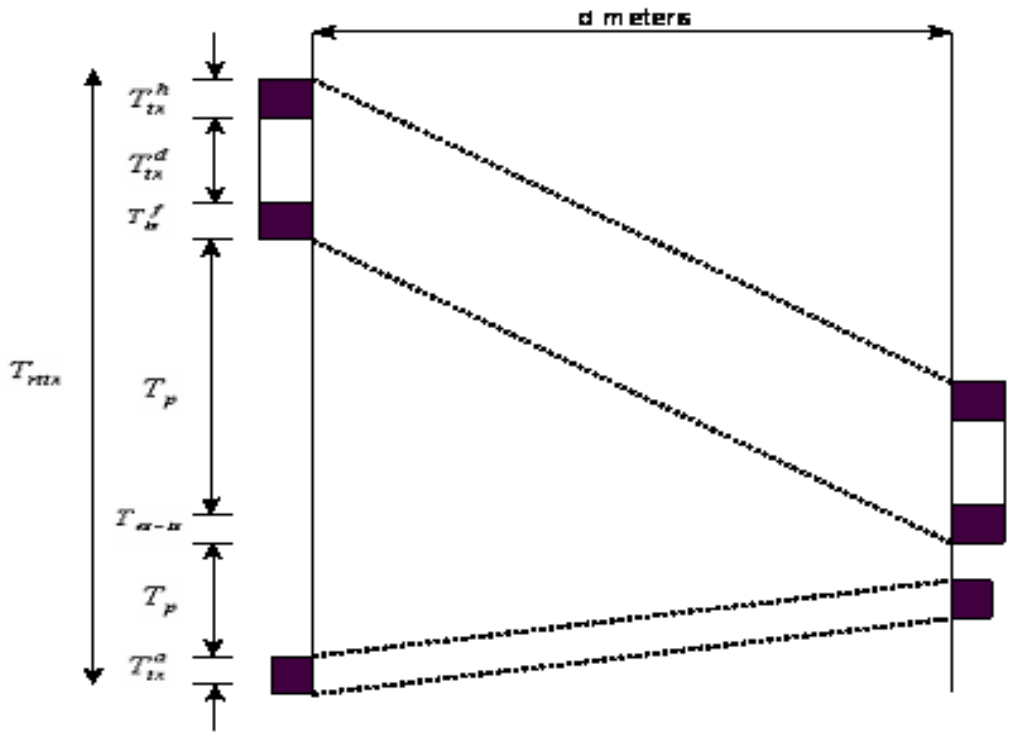

Figure 4(b)

Figure 4. Data Packet Transmission Model 
The cluster head is selected based on the signal strength, buffer length, distance between sensor nodes and sink node.

\subsection{Cluster Forming}

After cluster heads have been elected the other associate nodes will determine its own cluster based on the received signal strength of advertisements from cluster heads. Figure 5 shows the time line for the round of RROCH protocol.

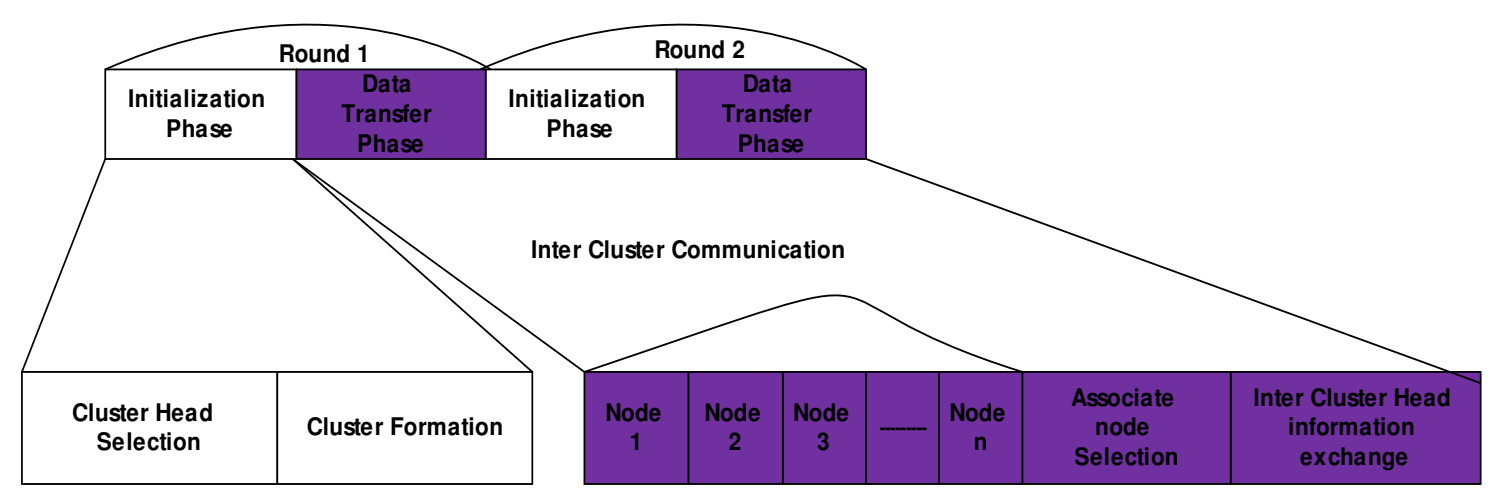

Figure 5. Time line of RROCH Protocol

\subsection{Intra-cluster Communication}

In the initialization phase, all the nodes except the cluster heads (CHs) generate $l$ bits sensing data with a transmission probability $P$ in different time slots of each round. The nodes within a cluster will exchange their information according to a OFDMA schedule set up by cluster head. We use the information exchange, which is a type of all-to-all communication, so that cluster heads do not send merged data to every associate node but only need to assign those nodes. The traditional methods to solve the intra cluster information exchange problem are flooding, relaying or routing. In RROCH protocol, we use network coding to reduce energy consumption and reduce the end to end delay.

Associate Nodes Selection in each round, associate node's number $m_{n}$ is decided by the threshold

$\alpha=\frac{E_{r e m}}{P_{t x-m}}$

$E_{r e m}$ is the remaining energy of each node and $P_{t x-m}$ is the transmission power for a member node sending 1 bit data to the sink node. All the selected member nodes have the largest value of $\alpha$ are assigned in a cluster.

To solve the intra-cluster communication problem, there are number of methods: flooding, routing, relaying and network coding. 


\subsubsection{Flooding}

Flooding is an old technique [14] that can also be used for routing in under water acoustic wireless sensor networks. In flooding, each node receiving a packet repeats it by broadcasting, unless a maximum number of hops for the packet are reached. Flooding is a reactive technique, and it does not require costly topology maintenance and complex route discovery algorithms. On the other hand, the main disadvantage of flooding algorithm is high energy consumption levels, which is an extremely important factor in under water acoustic wireless sensor networks.

\subsubsection{Random Routing}

In random routing each node does not know the global information about the cluster and can only communicate with its neighbors. In Figure 6, assume node $N_{1}$ wants to send information to node $N_{5}$, it does not know $N_{1} \rightarrow N_{6} \rightarrow N_{5}$ is the shortest path, and maybe it chooses the path: $N_{1} \rightarrow N_{2} \rightarrow N_{3} \rightarrow N_{4} \rightarrow N_{5}$. Without loss of generality, we assume the probability which path is chosen is 0.5 . Thus the average transmission number for node $N_{1}$ to deliver its information to any node in cluster is $\frac{n}{2}$.

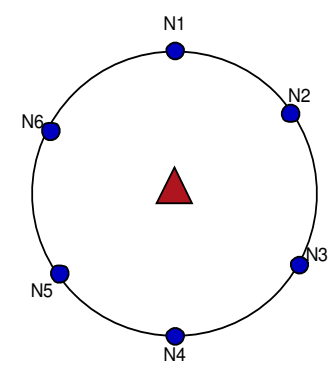

(a) Circular Topology

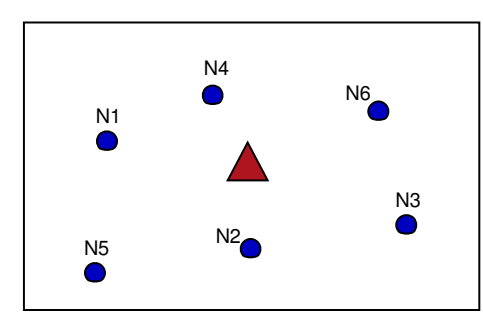

(b) Random Topology

Figure 6. Cluster Model for Intra-cluster Information Exchange

Consequently, the transmission number of one Node to spread its information to all the others is $\frac{n^{2}}{2}$ and $O\left(T_{\text {routing }}\right)=O\left(\frac{n^{3}}{2}\right)$. In random topology, it is difficult to calculate the accurate transmission number in random routing way. But we can estimate the average transmission hop is $O(\sqrt{n})$, so $O\left(T_{\text {routing }}\right)=O\left(\sqrt[n^{2}]{n}\right)$.

\subsubsection{Network Coding Algorithm}

In this algorithm, every node in the cluster needs to broadcasts the information only once and the cluster head just need broadcasts $\frac{n}{2}$ coded packets. Thus, the number of transmission of our network coding algorithm is $N C=n+\frac{n}{2}=\frac{3}{2} n$.Each node just sends own information directly to the cluster head without knowing any other information such as topology geography, etc. 
International Journal of Network Security \& Its Applications (IJNSA), Vol.4, No.3, May 2012
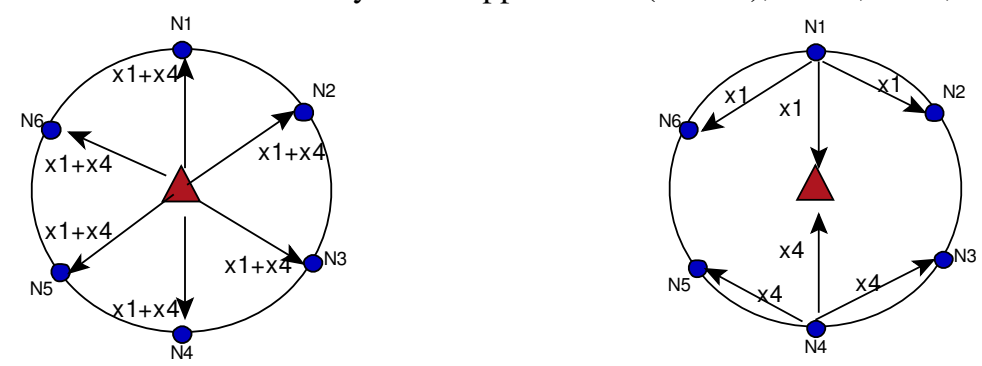

Figure 7. Network Coding Algorithm for Intra-cluster Information Exchange

By using network code algorithm the cluster head will at most broadcast extra $\frac{n}{2}$ packets in case that some nodes have not sufficient information to decode the packets. For example, in Fig. if node $N_{6}$ is out of the transmission range of node $N_{1}$ and $N_{4}$, consequently $N_{6}$ will not obtain $\mathrm{x} 1$ and $\mathrm{x} 4$, but only receive $x 1 \oplus x 4 \mathrm{x} 1$ from cluster head. Therefore, node $N_{6}$ cannot decode the information by itself. Thus the cluster head has to broadcast $\mathrm{x} 1$ or $\mathrm{x} 4$ to those nodes which have not efficient information to decode the incoming information. Thus, $\frac{3}{2} n \leq T_{N C} \leq 2 n=T_{\text {relay }}$.

\subsection{Inter Cluster Head communication}

Once the Cluster Head collects the information from its Associate nodes it has to transmit the information to Sink Node through Cluster Heads. OFDMA protocol is used for Inter Cluster Head communication. Orthogonal Frequency Division Multiple Access (OFDMA) is one of the recently proposed methods. The main feature of the OFDMA protocol is its robustness to multipath fading. OFDMA is a special case of multicarrier modulation (MCM) in which multiple user symbols are transmitted simultaneously using different subcarriers with overlapping frequency bands that are mutually orthogonal. It is the most promising technology that can deliver a wireless acoustic signal much farther with much less interference than competing technologies due to the orthogonality of the subcarriers. Orthogonal Frequency Division Multiplexing (OFDM) is a multiplexing technique that subdivides the available bandwidth into multiple orthogonal frequency sub-carriers. The data stream is divided into several parallel sub-streams of reduced data rate and each sub-stream is transmitted on a separate orthogonal sub-carrier. This reduces the occurrences of collision in transmission phase.

\section{Performance Analysis}

\subsection{Packet Delivery Ratio}

This is the ratio of packets received by sinks to the total number of packets generated by the network to send information. Packet Delivery Ratio as a function of number of Constant Bit error Rate connection. The plot infers that delivery ratio decreases as the number of connections increase. The maximum delivery ratio for Heed is just above $7 \%$ and decreases further for heavy traffic conditions.

$$
\text { PacketDeliveryRatio }=\frac{\text { Packets } \text { Re ceived by the Sink }}{\text { Packets generated through the network }}
$$


The higher packet delivery ratio is the better reliability of the network.

\subsection{Average End-to-end Delay}

End-to-end delay is the time require by packet to traverse from a source to the receiver. The end-to-end delay is averaged for all the received packets over the total simulation period.

\subsection{Throughput}

Throughput refers to the amount of data successfully transferred from a sender to a receiver in a given time, usually measured in bits or bytes per second. It is affected by many factors, for example, the efficiency of collision avoidance, control overhead, channel utilization, and latency. Like latency, the importance of throughput depends on different applications.

\subsection{Energy Consumption}

The main objective of the routing protocols is efficient delivery of information between sensor nodes and the sink. Also sensor node's lifetime heavily depends on the powered battery and they will use up their limited energy resource during sensing, processing and communication process. Especially, the process of communication consumes a significant amount of energy. So, energy efficient routing protocols and algorithms need to be carefully designed

\section{Simulation Scenario}

The objective of this paper is to analyze the performance of existing routing protocols in underwater acoustic sensor networks. The simulations are conducted using ns-2 [ref]. The traffic scenarios have constant bit rate (CBR) connections with randomly selected sources and sinks. In our simulation scenarios, nodes are deployed randomly over an area of $100 \mathrm{~m} x 100 \mathrm{~m}$ with an initial energy level of 10 Kjoules. To evaluate exist routing protocols simulations are done with varying traffic conditions by increasing up to 5 CBR connections, different number of nodes and by taking sensor nodes up to a depth of 100 meters. The bandwidth of the data channel was set to $4 \mathrm{kbps}$. Each data message packet was 512 bytes long and the surface sink was set at the center. Based on the energy consumption model presented in Figure 2, the optimum numbers of clusters are calculated. To evaluate and compare the performance of RROCH with LEACH, LEACH-M and HMR-LEACH in the heterogeneous UWASN, we have conducted simulations for two scenarios: first, a network with 100 nodes deployed over an area of size $100 \times 100$ square meter shown in Figure 8 and Figure 9, we denote a normal node with $(\mathrm{o})$, and the sink node $(\Delta)$.

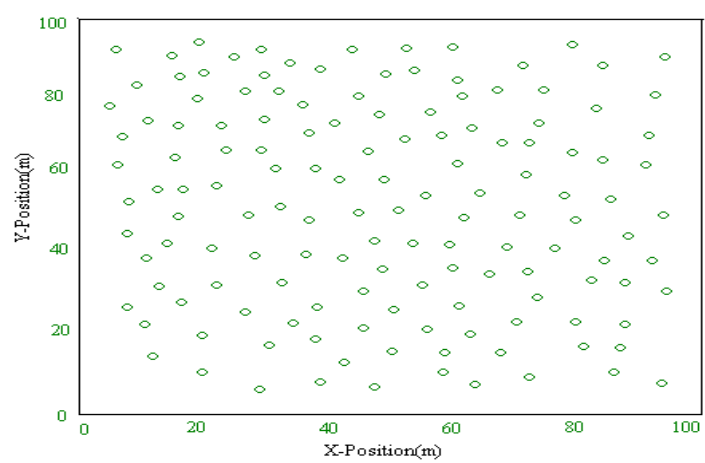

Figure 8. Random Deployments of Sensor Nodes 
International Journal of Network Security \& Its Applications (IJNSA), Vol.4, No.3, May 2012

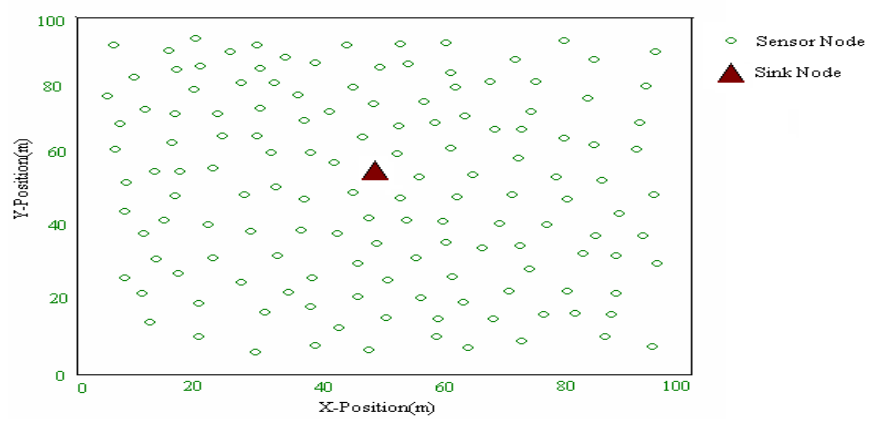

Figure 9. Random Deployment of Sensor nodes with Sink Node

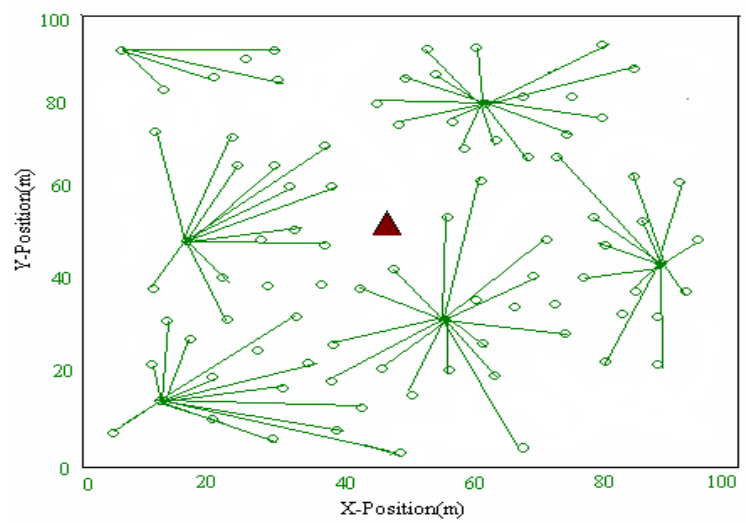

Figure 10. Cluster Head Selection based on energy Level

\subsection{Channel Utilization Efficiency}

Channel utilization refers to the bandwidth utilization for effective communication. Due to limited bandwidth, a routing protocol should make use of the bandwidth as efficiently as possible. From equation (16) and (17) we analyze the channel efficiency of the network. To find the channel efficiency for under water environment we set the speed of sound in water to $1500 \mathrm{~m} / \mathrm{sec}$ and the transmission rate to $50 \mathrm{Kbps}$. Figure 11 refers to transmissions without forward error correction $L^{f}=0$.From the plot we know that the channel efficiency goes down abruptly with increasing distance with respect to varying packet size.

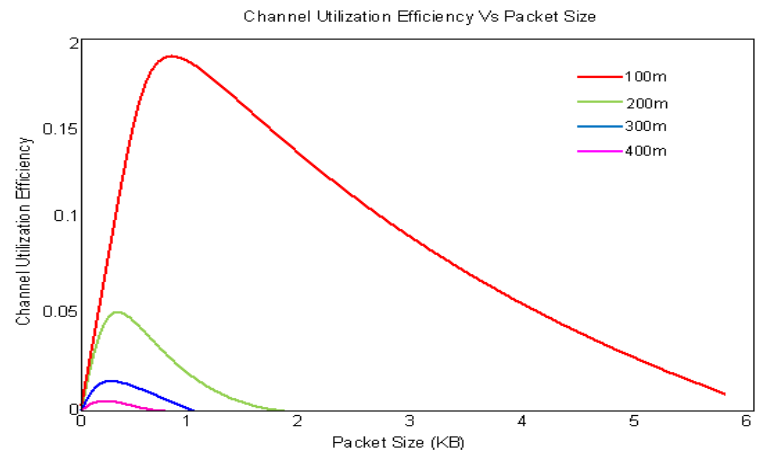

Figure 11 Underwater Channel Utilization Efficiency Vs Packet Size based on Various Distance 
International Journal of Network Security \& Its Applications (IJNSA), Vol.4, No.3, May 2012

\subsection{Packet Delivery Ratio}

\subsubsection{Based on CBR Connections}

Packet delivery ratio as a function of number of CBR connections is shown in Figure12. It is observed that packet delivery ratio decreases as the number of connections increase. The maximum delivery ratio for LEACH protocol is just above 6 percent and decreases further for heavy traffic conditions. HMR-LEACH shows a sharp decline as CBR connection is increased from 100 percent to just 2 percent. LEACH-M has the best overall delivery ratio. Even at high traffic conditions LEACH-M shows 8 times more delivery ratio than RROCH and LEACH. RROCH protocol performs better than LEACH-M in case of lesser number of connections with a packet delivery ratio of up to 90 percent but shows a degraded performance with a delivery ratio of less than 2 percent for the scenario with highest CBR traffic.

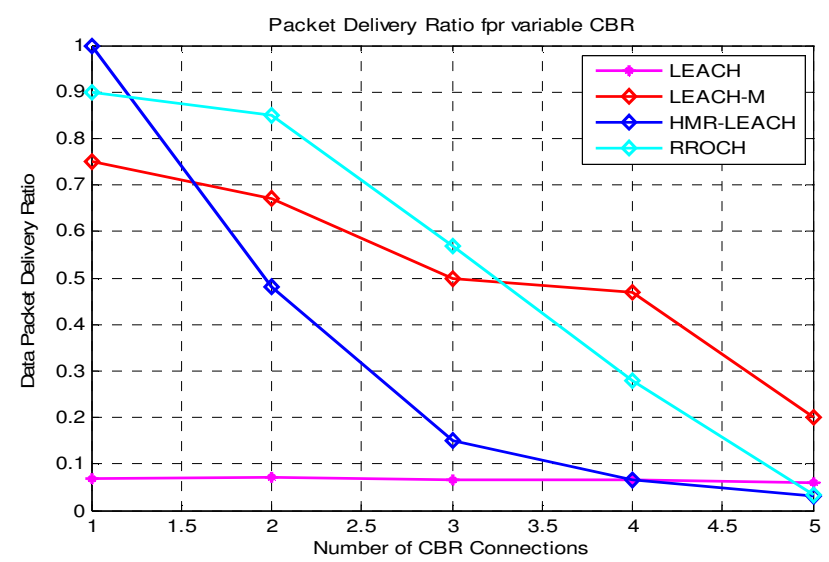

Figure 12. Packet Delivery Ratio based on Various CBR Connections

\subsubsection{Based on Number of Nodes}

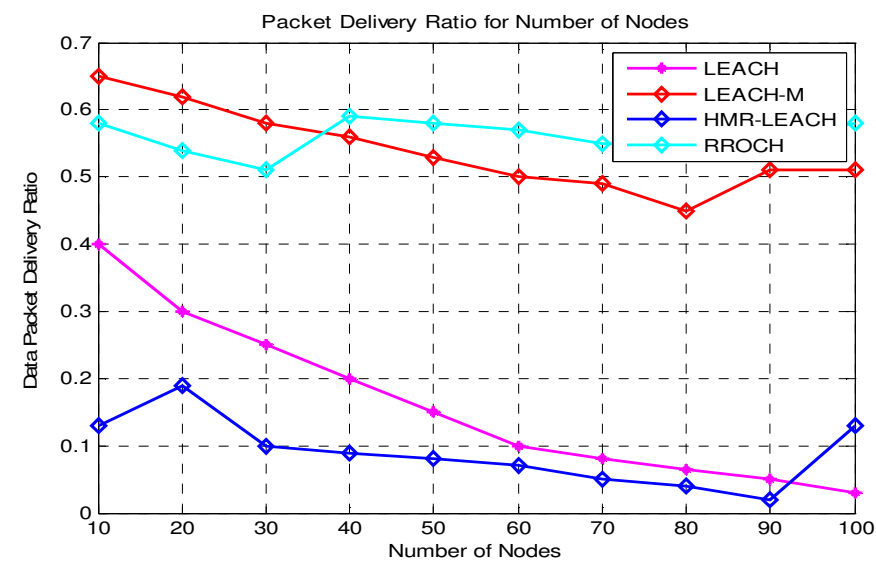

Figure 13. Packet Delivery Ratio based on Number of Nodes 
Packet delivery ratio vs. different number of nodes is shown in Figure13. RROCH protocol has the most stable packet delivery ratio. RROCH protocol has a standard deviation of 1.03 for packet delivery ratio and LEACH-M, HMR-LEACH and LEACH have standard deviations of 5.95, 6.43 and 13.08mseconds respectively. Average delivery ratio is lowest for HMR-LEACH for all different number of nodes. Delivery ratio for LEACH decreases as the number of nodes increases. Both, RROCH and LEACH-M have higher delivery ratios than LEACH and HMR LEACH protocol.

\subsubsection{Based on Various Depths}

Figure 14 shows the packet delivery ratio based on various depth. From the plot it is clear that RROCH performs better than LEACH-M, LEACH and HMR-LEACH protocol at all depths as it has the highest data delivery ratio of more than 50 percent. Packet delivery ratio for RROCH protocol remains almost unaffected by the change in depth. LEACH-M has significantly high data delivery ratios than LEACH and HMR-LEACH with the maximum of 49 percent and minimum of 38 percent. HMR-LEACH performs worst at depths of $25 \mathrm{~m}$ and $100 \mathrm{~m}$ with a delivery ratio of almost zero and performed relatively better than LEACH at other depths.

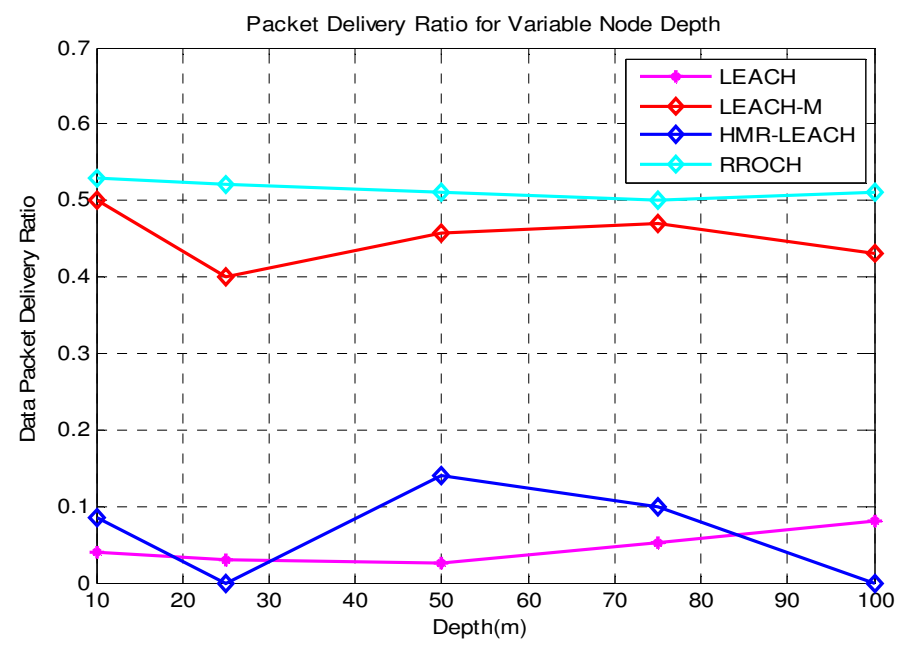

Figure 14. Packet Delivery Ratio based on Various Depth

\subsection{End- to- End Delay}

\subsubsection{Based on CBR Connections}

Figure 15 shows the end-to-end delay vs. number of CBR connections. HMR-LEACH protocol has the highest delay for all simulation scenarios. On average HMR-LEACH protocol has endto-end delay 5.6 times higher than LEACH protocol, 5 times higher than LEACH-M and 6 times higher than RROCH. It is observed that increasing the traffic affects the end-to-end delay. In case of RROCH, LEACH-M and LEACH protocol the end-to-end delay increases with the increase in traffic. However, HMR-LEACH protocol has the same trend up to $3 \mathrm{CBR}$ connections but with 5 CBR connections delay decreases by a factor of 3 . 
International Journal of Network Security \& Its Applications (IJNSA), Vol.4, No.3, May 2012

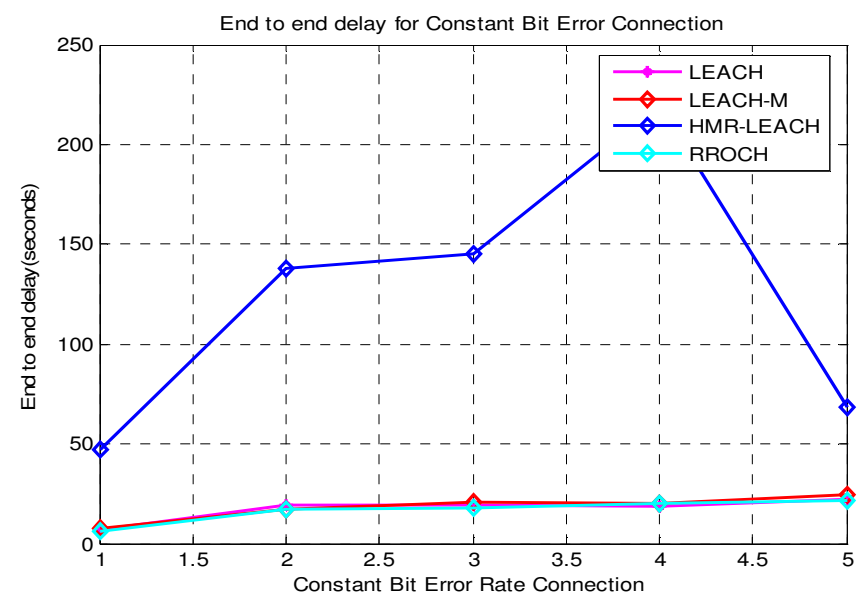

Figure 15 End-to-End Delay based on Various CBR Connections

\subsubsection{Based on Number of Nodes}

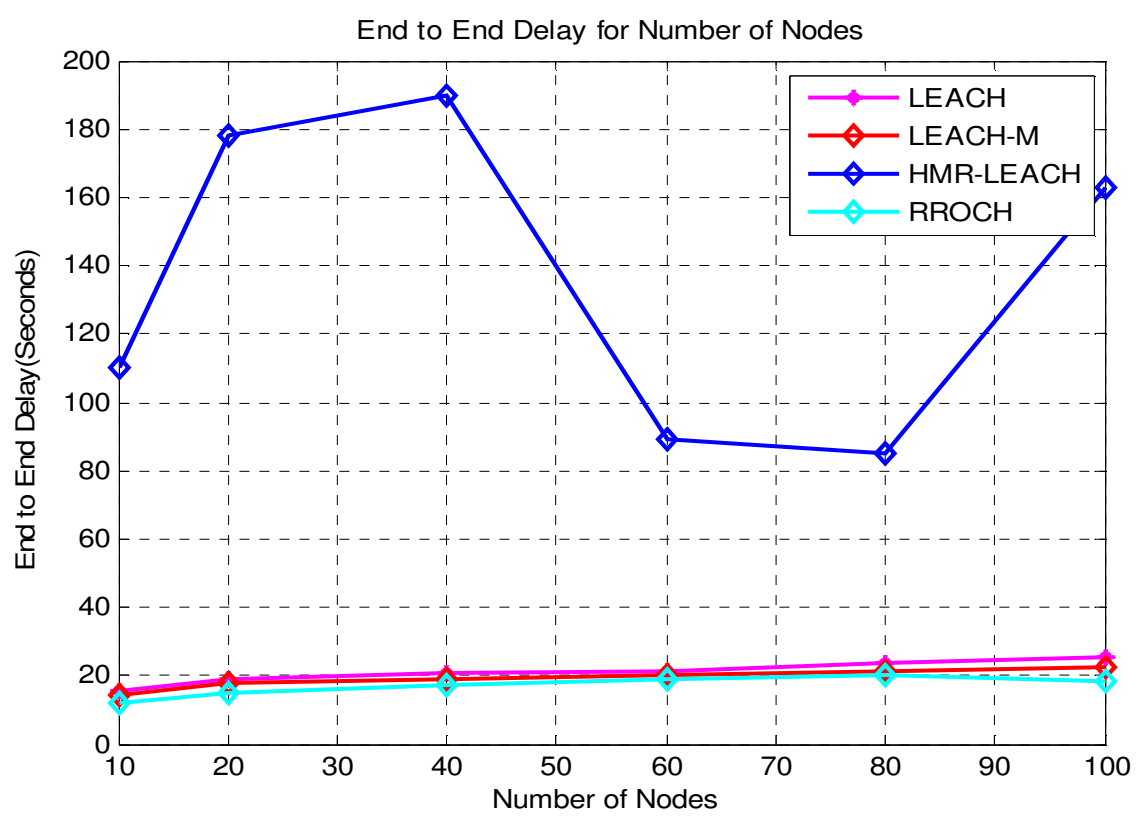

Figure 16. End-to-End Delay based on Number of Nodes

HMR-LEAH protocol again has the highest delay when analyzed with respect to number of nodes as shown in Figure16. RROCH, LEACH-M and LEACH protocols have comparable end-to-end delays but overall RROCH protocol has lowest delay. 
International Journal of Network Security \& Its Applications (IJNSA), Vol.4, No.3, May 2012

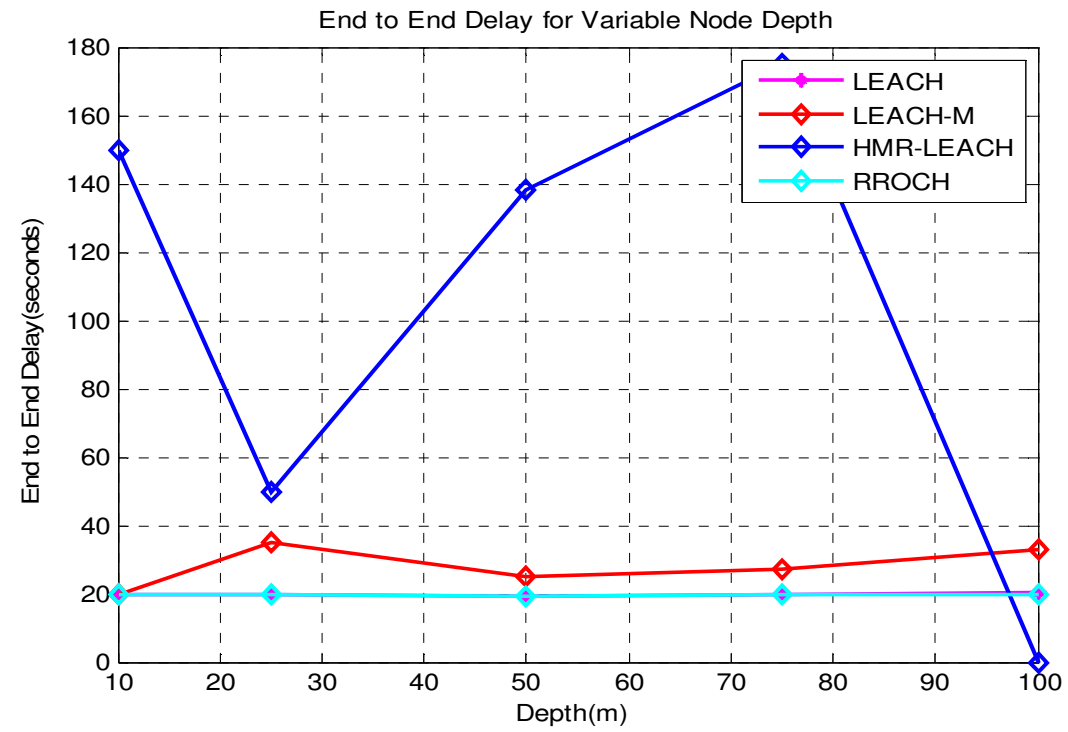

Figure 17. End-to-End Delay based on Various Depths

The effect of depth on end-to-end delay is shown in Figure 17. HMR-LEACH has the highest average end-to-end delay and is most affected by the change in depth. On average HMRLEACH has delay 9 times higher than RROCH, 7.5 times higher than LEACH-M and 7 times higher than LEACH when the depth is more than $70 \mathrm{~m}$. RROCH tends to maintain a steady endto-end delay and does not fluctuate much with the increase or decrease in depth.

\subsection{Throughput}

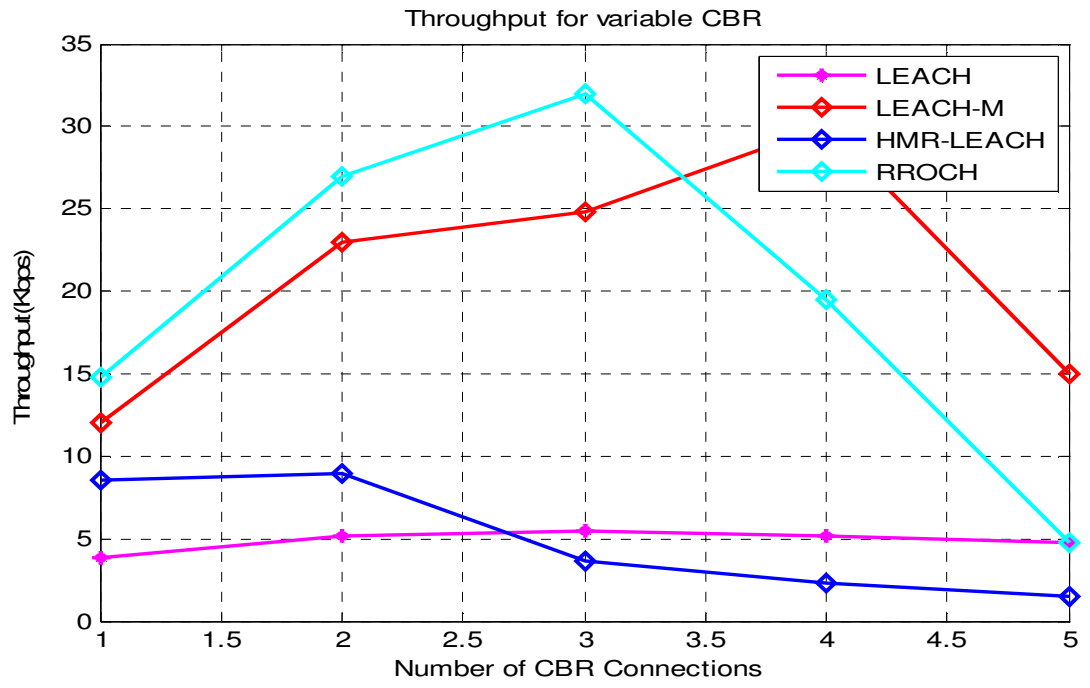

Figure 18. Throughput based on Various CBR Connections 
Figure 18 shows the throughput of RROCH protocol based on various CBR connections. From the plot we infer that up to certain point, throughput increases for RROCH protocol and LEACH-M as the traffic is increased. Although, LEACH has the lowest data rate but at the same time it has the most steady data rate and remains unaffected by the change in traffic conditions. In case of HMR-LEACH protocol is highly affected by the traffic conditions and drops to very low data rate for maximum traffic conditions. LEACH-M performs exceptionally well at higher traffic conditions by having a data rate almost 1.5 times higher than RROCH when CBR connection is 4 , which had highest data rate.

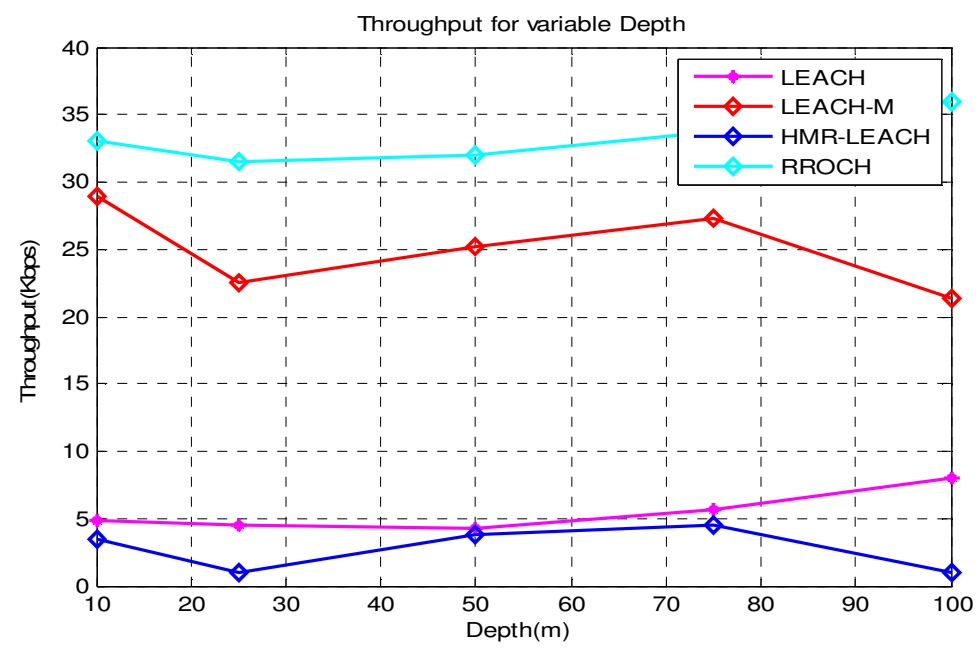

Figure 19. Throughput based on Various Depths

Throughput for LEACH-M and LEACH decreases with the increase in number of nodes, but after a certain number of nodes throughput improves slightly. This is expected by proactive protocols, as nodes are increased more and more routing packets are generated, thus affecting the actual throughput. Initially with lesser nodes this impact is higher and very visible, but afterward this effect gradually norms out and throughput takes a steady shape. This is shown in Figure 19. RROCH and HMR-LEACH show a fluctuating behavior with respect to throughput. This is due to their reactive nature, as numbers of nodes are changed the routes are changed which in turn affects the packet delivery ratio. A change in packet delivery ratio directly affects the average throughput of the nodes.

\subsection{Energy Consumption}

Average energy consumption of nodes for varying traffic conditions is shown in Figure 19. It is evident that LEACH-M protocol has the highest rate of energy consumption irrespective of the number of connections. This is because LEACH-M protocol has the highest routing overhead; meaning more send operations by the nodes which causes them to lose their energy sooner. It is also observed for all protocols that more energy is consumed as the traffic increases. RROCH and LEACH protocol has significant decrease in average energy consumption is observed when subjected to high traffic conditions because of increased route discovery messages. 


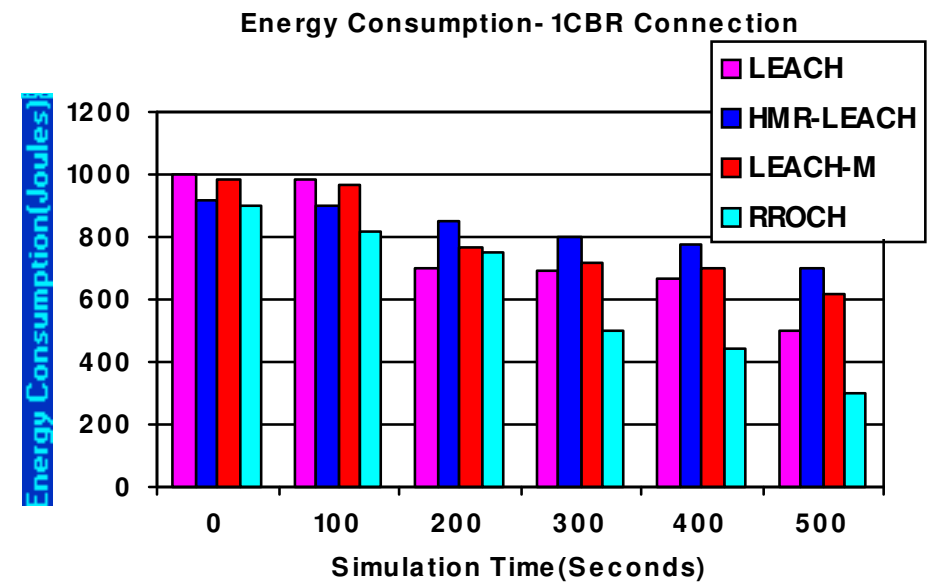

Figure 20 (a)

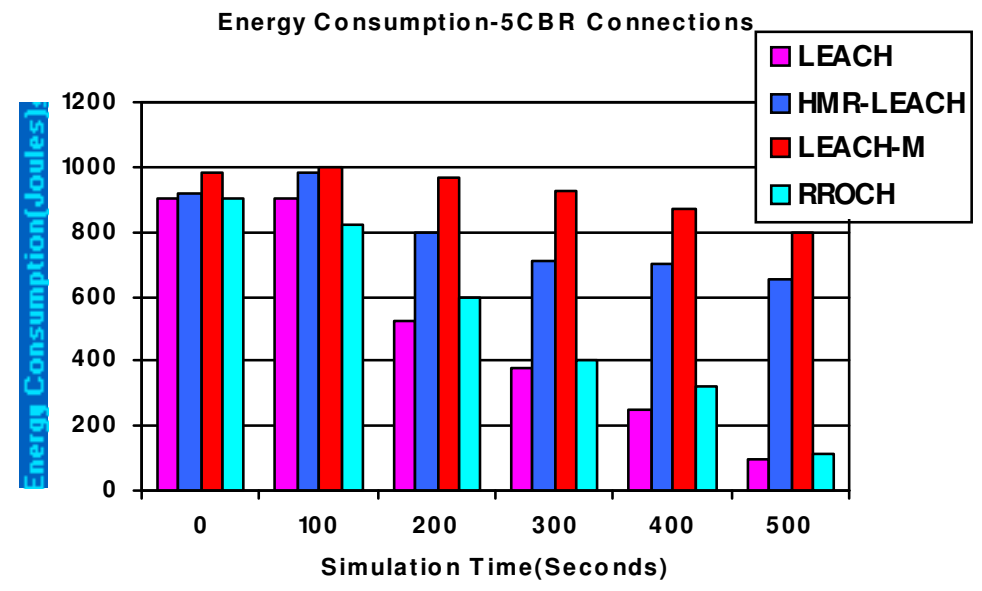

Figure $20(b)$

Figure 20. Energy Consumption based on Various CBR Connections

\section{Conclusions}

In this paper we made an effort to examine routing protocols in underwater acoustic sensor network environment. After analyzing the results, we concluded that HMR-LEACH protocol is not suitable for underwater networks due to its high rate of energy consumption. Energy consumption of sensor nodes is always a major concern in underwater networks. HMR-LEACH is also not suitable because of its high routing overhead. LEACH-M will also not be suitable because it shows very low packet delivery ratio and throughput. End-to-end delay and routing overhead sharply changes as number of connections, depth or nodes is changed. RROCH and LEACH on the other hand tend to perform better but both have some tradeoffs. RROCH has higher delivery ratio and throughput for lesser number of connections than LEACH but LEACH performs better at high traffic conditions. Both RROCH and LEACH have steady delivery ratios and end-to-end delays and do not fluctuate much with the change in number of 
International Journal of Network Security \& Its Applications (IJNSA), Vol.4, No.3, May 2012

nodes and depth. Overall delay is observed lesser in case of RROCH as compared to LEACH. Routing overhead is very little for RROCH with less traffic but increases multiplicatively with the increase in traffic. This also effects the energy consumption of the nodes. Nodes run out of energy sooner in high traffic conditions for RROCH. LEACH, more or less, has the same routing overhead for all traffic conditions. This may be a higher figure for lesser traffic conditions but as the traffic increases this is evened out. Energy consumption for LEACH is actually better than RROCH in higher traffic conditions. Based on the results, RROCH may be used for denser underwater networks but with less traffic and LEACH suits for higher traffic conditions with optimal number of nodes.

\section{REFERENCES}

[1] Ian F. Akyildiz , Dario Pompili, Tommaso Melodia,"Underwater acoustic sensor networks: research challenges", International Journal on Ad Hoc Networks, Vol 3, pp 257-279, 2005.

[2] Tracey ho and Desmond S.Lun, Published in the United States of America by Cambridge University Press, New York "Network Coding An Introduction”,2008.

[3] Dario Pompili, Tommaso Melodia and Ian F. Akyildiz, "A Distributed CDMA Medium Access Control for Underwater Acoustic Sensor Networks", Sixth Annual Mediterranean Ad Hoc Networking WorkShop, Corfu, Greece, June 12-15, 2007.

[4] Fatma Bouabdallah and Raouf Boutaba, “ A Distributed OFDMA Medium Access Control for Underwater Acoustic Sensors Networks" IEEE International Conference on Communications pp 1-5, ISBN: 978-1-61284-231-8, June 2011.

[5] Guangzhong Liu and Changye Wei, "A New Multipath Routing Protocol Based on Cluster for Underwater Acoustic Sensor Networks" IEEE National Natural Science Foundation pp 91-94, ISBN: 978-1-61284-771-9,July 2011.

[6] Guangsong Yang, Mingbo Xiao, En Cheng and Jing Zhang, "A Cluster-head Selection Scheme for Underwater Acoustic Sensor Networks" IEEE International Conference on Communications and Mobile Computing,pp 188-191, ISBN: 978-1-4244-6327-5, April 2010.

[7] Jinyong Cheon and Ho-Shin Cho, "A delay-tolerant OFDMA-based MAC Protocol for Underwater Acoustic Sensor Networks" IEEE International Conference on Communications pp 1-4, ISBN: 978-1-4577-0165-8,April 2011.

[8] Long-Shin Chen, Zong-Yi Lyu and Zeng-Wen Hong, "An Efficient Cluster Head Selection Strategy for Wireless Sensor network" IEEE Fifth conference on Genetic and Evolutionary Computing pp 37-40, ISBN: 978-1-4577-0817-6, August 2011.

[9] Dang Nguyen, Pascale Minet, Thomas Kunz and Louise Lamont, "New Findings on the Complexity of Cluster Head Selection Algorithms" IEEE International Symposium on World of Wireless, Mobile and Multimedia Networks pp 1-10, ISBN: 978-1-4577-0352-2, June 2011.

[10] H. N. Egbo, N. J. Hallin, P. L. Ray, M. O’Rourke, J.F. Frenzel and D. B. Edwards, “TokenBased Medium Access Control Solution for Underwater Acoustic Broadcast" IEEE BiloxiMarine Technology for our future goal: Global and Local Challenges pp1-9, ISBN: 978-1-42444960-6, October 2009.

[11] Borja Peleato and Milica Stojanovic, "Distance Aware Collision Avoidance Protocol for AdHoc Underwater Acoustic Sensor Networks", IEEE Communications Letters, Vol.11, Issue 12, pp 1025 - 1027 ,ISBN: 1089-7798, December 2007. 
International Journal of Network Security \& Its Applications (IJNSA), Vol.4, No.3, May 2012

[12] Yun Li; Nan Yu; Weiyi Zhang; Weiliang Zhao; Xiaohu You; Daneshmand, M, "Enhancing the performance of LEACH protocol in wireless sensor networks ",IEEE Conference on Computer Communications Workshops (INFOCOMS WKSHPS) pp 223 228, ISBN: 978- 1-4577-0249-5, April 2011.

[13] Guangzhong Liu, Changye Wei , "A new multi-path routing protocol based on cluster for underwater acoustic sensor networks ", International Conference on Multimedia Technology (ICMT) pp 91 - 94, ISBN: 978-1-61284-771-9, July 2011.

[14] Heikalabad, S.R. ; Firouz, N. ; Navin, A.H. ; Mirnia, M.K, "HEECH: Hybrid Energy Effective Clustering Hierarchical Protocol for Lifetime Prolonging in Wireless Sensor Networks", International Conference on Computational Intelligence and Communication Networks (CICN) pp 325 - 328, ISBN: 978-1-4244-8653-3, November 2010.

\section{Authors}

T.V.Padmavathy obtained her A.M.I.E (ECE) from the Institution of Engineers (India), M.C.A from University of Madras and M.E., degree from College of Engineering, Anna University in the years 1996, 2001 and 2004 respectively. She has 13 years of teaching experience. She is working as Assistant Professor in the Department of Electronics and Communication Engineering, Chennai. She is presently a research scholar in Anna University. She has published over 19 research papers in National/International Journal and conference proceedings. She is a member of IEEE, IETE and ISTE. Her area of interest is Wireless Sensor Networks and Embedded Systems.

Gayathri.V, educated from Maharishi Vidya Madir upto class XII, played an important role in all school activities, especially specializing in French, obtaining state rank and received awards. She is good at volleyball and has represented her school in district level tournaments. She has a passion to play veena. She got ECE branch in RMKCET on merit and presently pursuing her final year with excellent performance throughout. She Won "Best Paper Award" for two papers in national conferences held at Vel Tech Engineering College and at Vel Srinivasa Engineering College. Her areas of interest are Wireless Sensor Networks and RFID.

Indhumathi.V, currently doing her Bachelor of Engineering in Electronics and Communication Engineering in R.M.K College of Engineering and Technology. She did her schooling in Vellayan Chettiyar Higher Secondary School. She won proficiency award for the year2009-2011 and got Distinction in Mathematics in S.S.L.C. Her field of interest includes wireless sensor networks and radio frequency engineering.

Karthika.G, currently doing her Bachelor of Engineering in Electronics and Communication Engineering in R.M.K College of Engineering and Technology. She did her schooling in Velammal Matriculation Higher Secondary School. Her field of interest includes Networking and security.
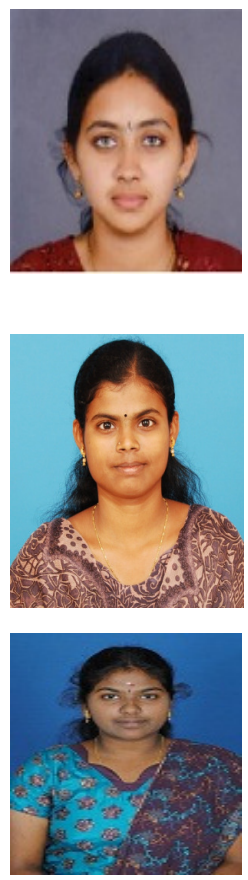